\title{
ALGEBRAS WITH POLYNOMIAL IDENTITIES AND BERGMAN POLYNOMIALS *
}

\author{
Tsetska Grigorova Rashkova \\ University of Rousse "A.Kanchev" \\ E-mail: tcetcka@ami.ru.acad.bg
}

Abstract. The talk is an introduction to the theory of algebras with polynomial identities. It stresses on matrix algebras and polynomial identities for them. The notion of Bergman polynomials is introduced. Such types of polynomials are investigated being identities for algebras with symplectic involution. In the Lie case more information is given for Bergman polynomials as Lie identities for the considered algebras.

\section{ALGEBRAS WITH POLYNOMIAL IDENTITIES}

We fix a countably infinite set $X=\left\{x_{1}, x_{2}, \ldots\right\}$ and consider a field $K$ of characteristic zero. We work in the algebra $K\langle X\rangle$ with basis the set of all words

$$
x_{i_{1}} \ldots x_{i_{k}}, x_{i_{j}} \in X
$$

and multiplication defined by

$$
\left(x_{i_{1}} \ldots x_{i_{m}}\right)\left(x_{j_{1}} \ldots x_{j_{n}}\right)=x_{i_{1}} \ldots x_{i_{m}} x_{j_{1}} \ldots x_{j_{n}} .
$$

Definition 1. (i)Let $f=f\left(x_{1}, \ldots, x_{n}\right) \in K\langle X\rangle$ and let $R$ be an associative algebra. We say that $f=0$ is a polynomial

*Partially supported by Grant MM1503/2005 of the Bulgarian Foundation for Scientific Research. 
identity for $R$ if

$$
f\left(r_{1}, \ldots, r_{n}\right)=0, \forall r_{1}, \ldots, r_{n} \in R .
$$

(ii) If the associative algebra $R$ satisfies a non-trivial polynomial identity $f$ (i.e. $f$ is a nonzero element from $K\langle X\rangle$ ), we call $R$ a PI-algebra.

It could be shown that $f \in K\langle X\rangle$ is a polynomial identity for $R$ if and only if $f$ is in the kernel of all homomorphisms $K\langle X\rangle \rightarrow R$.

We give some examples:

(i) The algebra $R$ is commutative if and only if it satisfies the polynomial identity

$$
\left[x_{2}, x_{2}\right]=x_{1} x_{2}-x_{2} x_{1}=0 .
$$

(ii) Let $R$ be a finite dimensional associative algebra and let $\operatorname{dim} R<n$. Then $R$ satisfies the standard identity of degree $n$

$$
s_{n}\left(x_{1}, \ldots, x_{n}\right)=\sum_{\sigma \in S_{n}}(\operatorname{sign} \sigma) x_{\sigma(1)} \ldots x_{\sigma(n)}=0,
$$

where $S_{n}$ is the symmetric group of degree $n$. The algebra $R$ also satisfies the Capelli identity

$d_{n}\left(x_{1}, \ldots, x_{n} ; y_{1}, \ldots, y_{n+1}\right)=\sum_{\sigma \in S_{n}}(\operatorname{sign} \sigma) y_{1} x_{\sigma(1)} y_{2} \ldots y_{n} x_{\sigma(n)} y_{n+1}=0$

(iii) Let $M_{2}(K)$ be the $2 \times 2$ matrix algebra. It satisfies the following polynomial identities:

1. The standard identity $s_{4}\left(x_{1}, x_{2}, x_{3}, x_{4}\right)=0$.

2. The Hall identity $\left[\left[x_{1}, x_{2}\right]^{2}, x_{3}\right]=0$.

The algebra $M_{2}(K)$ does not satisfy the Capelli identity $d_{4}=0$ and the standard identity $s_{3}=0$.

(iv) The $n \times n$ matrix algebra $M_{n}(K)$ satisfies the identity of algebraicity 


$$
\begin{gathered}
d_{n+1}\left(1, x, x^{2}, \ldots, x^{n} ; 1, y_{1}, \ldots, y_{n}, 1\right) \\
=\sum_{\sigma \in S_{n+1}}(\operatorname{sign} \sigma) x^{\sigma(0)} y_{1} x^{\sigma(1)} y_{2} \ldots y_{n} x^{\sigma(n)}=0,
\end{gathered}
$$

where the symmetric group $S_{n+1}$ acts on $\{0,1, \ldots, n\}$, and the identity

$$
s_{n}\left([x, y]\left[x^{2}, y\right], \ldots,\left[x^{n}, y\right]\right)=0 .
$$

(v) Let $U_{n}(K)$ be the algebra of $n \times n$ upper triangular matrices. It satisfies the identity

$$
\left[x_{1}, x_{2}\right] \ldots\left[x_{2 n-1}, x_{2 n}\right]=0 .
$$

Some important properties of associative algebras are expressed in the language of polynomial identities. We have seen this for the commutativity. Others examples come from nonunitary algebras. The algebra $R$ is nil of bounded index if there exists an $n \in N$ such that $x^{n}=0$ is an identity for $R$; the algebra $R$ is nilpotent of class $\leq n$ if $x_{1} \ldots x_{n}=0$ for $R$.

More details could be found in [4].

It turns out that the class of all PI-algebras has a good structure and combinatorial properties similar to those of the commutative and the finite dimensional algebras.

\section{BERGMAN POLYNOMIALS IN ASSOCIATIVE PI-ALGEBRAS}

We define a class of homogeneous associative polynomials, called Bergman polynomials [1]. These are homogeneous and multilinear in $y_{1}, \ldots, y_{n}$ polynomials $f\left(x, y_{1}, \ldots, y_{n}\right)$ from the free associative algebra $K\left\langle x, y_{1}, \ldots, y_{n}\right\rangle$ which can be written as

$$
f\left(x, y_{1}, \ldots, y_{n}\right)=\sum_{i=\left(i_{1}, \ldots, i_{n}\right) \in S_{n}} v\left(g_{i}\right)\left(x, y_{i_{1}}, \ldots, y_{i_{n}}\right),
$$

where $g_{i} \in K\left[t_{1}, \ldots, t_{n+1}\right]$ are homogeneous polynomials in commuting variables

$$
g_{i}\left(t_{1}, \ldots, t_{n+1}\right)=\sum \alpha_{p} t_{1}^{p_{1}} \ldots t_{n+1}^{p_{n+1}}
$$


and

(2) $v\left(g_{i}\right)=v\left(g_{i}\right)\left(x, y_{i_{1}}, \ldots, y_{i_{n}}\right)=\sum \alpha_{p} x^{p_{1}} y_{i_{1}} \ldots x^{p_{n}} y_{i_{n}} x^{p_{n+1}}$.

The following theorem of Bergman shows how one could investigate PI-algebras using commutative theory approach.

Proposition 1 [1, Section 6, (27)]

(i) The polynomial $v\left(g_{i}\right)$ from (2) is an identity for $M_{n}(K)$ if and only if

$$
\prod_{1 \leq p<q \leq n+1}\left(t_{p}-t_{q}\right)
$$

divides $g_{i}\left(t_{1}, \ldots, t_{n+1}\right)$ for all $i=\left(i_{1}, \ldots, i_{n}\right)$.

(ii) The polynomial $f\left(x, y_{1}, \ldots, y_{n}\right)$ from (1) is an identity for $M_{n}(K)$ if and only if every summand $v\left(g_{i}\right)$ is also an identity for $M_{n}(K)$.

If the algebra has some additional properties analogues of Bergman theorem could be formulated. These properties are concerned with the existence of involutions (i.e. antiautomorphisms of second order) in the considered algebras.

We recall that in the matrix algebra $M_{2 n}(K, *)$ the symplectic involution $*$ is defined by

$$
\left(\begin{array}{cc}
A & B \\
C & D
\end{array}\right)^{*}=\left(\begin{array}{cc}
D^{t} & -B^{t} \\
-C^{t} & A^{t}
\end{array}\right)
$$

where $A, B, C, D$ are $n \times n$ matrices and $t$ is the usual transpose.

For an algebra $R$ with involution $*$ we have $(R, *)=R^{+} \oplus R^{-}$, where $R^{+}=\left\{r \in R \mid r^{*}=r\right\}$ and $R^{+}=$ $\left\{r \in R \mid r^{*}=-r\right\}$.

We call $f\left(x_{1}, \ldots, x_{n}\right) \in K\langle X\rangle$ a $*$-polynomial identity for $(R, *)$ in symmetric variables if $f\left(r_{1}^{+}, \ldots, r_{n}^{+}\right)=0$ for all $r_{1}^{+}, \ldots, r_{n}^{+} \in R^{+}$. Analogously $f\left(x_{1}, \ldots, x_{s}\right) \in K\langle X\rangle$ is a *polynomial identity for $(R, *)$ in skew-symmetric variables if $f\left(r_{1}^{-}, \ldots, r_{s}^{-}\right)=0$ for all $r_{1}^{-}, \ldots, r_{s}^{-} \in R^{-}$. 
The algebra $R^{+}$is a Jordan algebra with respect to the multiplication $r_{1}^{+} \circ r_{2}^{+}=r_{1}^{+} r_{2}^{+}+r_{2}^{+} r_{1}^{+} ; r_{1}^{+}, r_{2}^{+} \in R^{+}$and the identities in symmetric variables are weak polynomial identities for the pair $\left(R, R^{+}\right)$.

Similarly, the algebra $R^{-}$is a Lie algebra with respect to the new multiplication $\left[r_{1}^{-}, r_{2}^{-}\right]=r_{1}^{-} r_{2}^{-}-r_{2}^{-} r_{1}^{-} ; r_{1}^{-}, r_{2}^{-} \in R^{-}$and the identities in skew-symmetric variables for $(R, *)$ are weak polynomial identities for the pair $\left(R, R^{-}\right)$.

In order to state the next result we introduce the following natation, namely

$$
g_{2 n, 0}=\prod_{\substack{1 \leq p<q \leq n+1 \\(p, q) \neq(1, n+1)}}\left(t_{p}^{2}-t_{q}^{2}\right)\left(t_{1}-t_{n+1}\right) .
$$

Proposition 2 [9, Theorem 3] Considered in $M_{2 n}(K, *)$, the polynomial $f$ from (1) satisfies $f\left(a, r_{1}, \ldots, r_{n}\right)=0$ for any skew-symmetric matrix $a$ and all matrices $r_{1}, \ldots, r_{n}$ if and only if $\left(t_{1}+t_{n+1}\right) g_{2 n, 0}$ divides the polynomials $g_{i}\left(t_{1}, \ldots, t_{n+1}\right)$ for all $i=\left(i_{1}, \ldots, i_{n}\right)$.

The sufficient condition of this proposition could be improved.

Proposition 3 [8, Theorem 1] Let the polynomial (1) be a *identity in skew-symmetric variables for the algebra $M_{2 n}(K, *)$. Then the polynomial $g_{2 n, 0}$ divides the polynomials $g_{i}$ from (2) for all $i=\left(i_{1}, \ldots, i_{n}\right)$.

Some other results are the following:

Proposition 4 [8, Proposition 3] The linearization in y of the standard polynomial $S_{3}\left(\left[x^{3}, y\right],\left[x^{2}, y\right],[x, y]\right)$ is an identity in symmetric variables for $M_{6}(K, *)$ of minimal degree.

Proposition 5 [5, Theorem 3] A polynomial $f$ is a Bergman type identity in skew-symmetric variables for $M_{4}(K, *)$ if and 
only if it has the form

$$
\begin{aligned}
f & =\alpha\left(v\left(g_{1}\right)\left(x, y_{1}, y_{2}\right)+v\left(g_{2}\right)\left(x, y_{2}, y_{1}\right)\right) \\
& +\beta v\left(g_{3}\right)\left(x, y_{1}, y_{2}\right)+\gamma v\left(g_{4}\right)\left(x, y_{2}, y_{1}\right),
\end{aligned}
$$

where

1. $g_{1}=g_{4,0} \prod_{i}\left(a_{i} t_{1}+b_{i} t_{2}+c_{i} t_{3}\right)$,

$g_{2}=g_{4,0} \Pi_{i}\left(-c_{i} t_{1}-b_{i} t_{2}-a_{i} t_{3}\right)$

and $t_{1}+t_{3}$ is not a factor of the polynomials $g_{1}$ and $g_{2}$;

2. The polynomial $\left(t_{1}+t_{3}\right) g_{4,0}$ divides $g_{3}$ and $g_{4}$ and

3. The identity $v\left(g_{1}\right)\left(x, y_{1}, y_{2}\right)+v\left(g_{2}\right)\left(x, y_{2}, y_{1}\right)=0$ follows from the identity

$f_{0}\left(x, y_{1}, y_{2}\right)=\sum_{\sigma \in S_{2}} v\left(g_{4,0}\right)\left(x, y_{\sigma(1)}, y_{\sigma(2)}\right)=0$.

We are able to formulate and prove a result for $M_{2 n}(K, *)$ generalizing the "only if" part of Proposition 5 for $n=2$.

Theorem 1 [8, Theorem 3] For $n \equiv 2,3(\bmod 4)$ every Bergman type polynomial of degree $k$ of the form

$$
f=\alpha \sum_{i} v\left(g_{i}\right)\left(x, y_{i_{1}}, \ldots, y_{i_{n}}\right)+\beta \sum_{j} v\left(g_{j}\right)\left(x, y_{j_{1}}, \ldots, y_{j_{n}}\right)
$$

is a $*$-identity in skew-symmetric variables for $M_{2 n}(K, *)$, where 1. $g_{i}=g_{2 n, 0} \prod_{l=1}^{k-n^{2}-2 n+1} \sum_{m=1}^{n} a_{i, m}^{(l)} t_{m}$,

$g_{i+\frac{n !}{2}}=g_{2 n, 0} \prod_{l=1}^{k-n^{2}-2 n+1}\left(-\sum_{m=1}^{n} a_{i, n+1-m}^{(l)} t_{m}\right), i=1, \ldots, \frac{n !}{2}$

and $t_{1}+t_{n+1}$ is not a factor of these polynomials;

2. The polynomial $\left(t_{1}+t_{n+1}\right) g_{2 n, 0}$ divides $g_{j}$ and

3. The identity $\sum v\left(g_{i}\right)\left(x, y_{i_{1}}, \ldots,, y_{i_{n}}\right)=0$ follows from the identity

$$
v\left(g_{2 n, 0}\right)\left(x, y_{i_{1}}, y_{i_{2}}, \ldots, y_{i_{n}}\right)+v\left(g_{2 n, 0}\right)\left(x, y_{i_{n}}, y_{i_{n-1}}, \ldots, y_{i_{1}}\right),
$$

for $\left(i_{1}, i_{2}, \ldots, i_{n}\right) \in S_{n}$. 


\section{LIE ALGEBRAS}

Starting with the free Lie algebra $L(X)$ we can define the notion of polynomial identity for a Lie algebra $G$. We give some examples:

(i) Let $G$ be the two-dimensional Lie algebra with basis as a vector space $\{a, b\}$ and multiplication $[a, b]=a$. The algebra $G$ satisfies the polynomial identity (namely the metabelian identity)

$$
\left[\left[x_{1}, x_{2}\right],\left[x_{3}, x_{4}\right]\right]=0 .
$$

(ii) If $G$ is a finite dimensional Lie algebra and $\operatorname{dim} G<n$, then $G$ satisfies the Lie standard identity of degree $n+1$ (but in $n$ skew-symmetric variables)

$$
x_{0} s_{n}\left(a d x_{1}, \ldots, a d x_{n}\right)=\sum_{\sigma \in S_{n}}(\operatorname{sign} \sigma)\left[x_{0}, x_{\sigma(1)}, \ldots, x_{\sigma(n)}\right]=0 .
$$

(iii) The Lie algebra $\left(U_{n}(K)\right)^{(-)}$of all upper triangular $n \times n$ matrices satisfies the identity

$$
\left[\left[x_{1}, x_{2}\right], \ldots,\left[x_{2 n-1}, x_{2 n}\right]\right]=0 .
$$

(iv) Let $W_{n}$ be the set of all derivations of the polynomial algebra in $n$ variables. (The linear operator $\delta$ of the vector space $K\left[x_{1}, \ldots, x_{n}\right]$ is a derivation if $\delta(u v)=\delta(u) v+u \delta(v), u, v \in$ $\left.K\left[x_{1}, \ldots, x_{n}\right].\right) W_{n}$ is a Lie algebra with respect to the operation $\left[\delta_{1}, \delta_{2}\right]$.

1. The algebra $W_{1}$ satisfies the Lie standard identity

$$
x_{o} s_{4}\left(a d x_{1}, a d x_{2}, a d x_{3}, a d x_{4}\right)=0 .
$$

2. The algebra $W_{n}$ satisfies some Lie standard identity.

As in the case of associative algebras, some classical properties and results for Lie algebras can be stated in the language of polynomial identities. A Lie algebra $G$ is abelian if it satisfies the identity $\left[x_{1}, x_{2}\right]=0$ meaning that $G$ has a trivial multiplication. The algebra $G$ is nilpotent of class $\leq n$ if it satisfies $\left[x_{1}, \ldots, x_{n}\right]=0$. The algebra $G$ is solvable of class $\leq n$ if it 
satisfies the identity $f_{n}=0$, where $f_{n}=f_{n}\left(x_{1}, \ldots, x_{2^{n}}\right)=0$, is defined inductively by

$$
\begin{gathered}
f_{1}\left(x_{1}, x_{2}\right)=\left[x_{1}, x_{2}\right], \\
f_{n}=\left[f_{n-1}\left(x_{1}, \ldots, x_{2^{n-1}}\right), f_{n-1}\left(x_{2^{n-1}+1}, \ldots, x_{2^{n}}\right)\right], n>1 .
\end{gathered}
$$

The solvable of class 2 Lie algebras are called metabelian. Any solvable finite dimensional Lie algebra satisfies the identity

$$
\left[\left[x_{1}, x_{2}\right], \ldots,\left[x_{2 n-1}, x_{2 n}\right]\right]=0
$$

for some positive $n$.

\section{BERGMAN POLYNOMIALS IN LIE ALGEBRAS}

It is a natural question to consider Bergman polynomials in Lie algebras as well. Working in the Lie algebra $s o(4, \mathrm{~K}, *)$ of the skew-symmetric to the symplectic involution $*$ variables of the matrix algebra of fourth order $M_{4}(K, *)$ we are interested in finding the minimal degree of these polynomials. In [6] using the Hall basis of the free algebra $L(X)$ for $X=\left\{x, y_{1}, y_{2}\right\}$ we consider the following elements of a given degree $k+2$ : $[y_{i_{1}}, \underbrace{x, \ldots, x}_{k}, y_{i_{2}}]$ and $\left[[y_{i_{1}}, \underbrace{x, \ldots, x}_{l}],[y_{i_{2}}, \underbrace{x, \ldots, x}_{k-l}]\right]$, where $\left(i_{1}, i_{2}\right)$ is any permutation on $\{1,2\}$ and $l=1, \ldots, k-1$.

The left normed commutators are written as elements of the free associative algebra $K\langle X\rangle$ and thus the commutative polynomials are uniquely defined.

For example for

$$
\begin{aligned}
f & =\left[y_{1}, x, y_{2}\right]=y_{1} x y_{2}-x y_{1} y_{2}-y_{2} y_{1} x+y_{2} x y_{1} \\
& =v\left(g_{1}\right)\left(x, y_{1}, y_{2}\right)+v\left(g_{2}\right)\left(x, y_{2}, y_{1}\right)
\end{aligned}
$$

we have $g_{1}=t_{2}-t_{1}$ and $g_{2}=-\left(t_{3}-t_{2}\right)$. 
For

$$
\begin{aligned}
f & =\left[\left[y_{1}, x\right],\left[y_{2}, x, x\right]\right]=y_{1} x y_{2} x^{2}-2 y_{1} x^{2} y_{2} x+y_{1} x^{3} y_{2} \\
& -x y_{1} y_{2} x^{2}+2 x y_{1} x y_{2} x-x y_{1} x^{2} y_{2}-y_{2} x^{2} y_{1} x+y_{2} x^{3} y_{1} \\
& +2 x y_{2} x y_{1} x-2 x y_{2} x^{2} y_{1}-x^{2} y_{2} y_{1} x+x^{2} y_{2} x y_{1} \\
& =v\left(g_{1}\right)\left(x, y_{1}, y_{2}\right)+v\left(g_{2}\right)\left(x, y_{2}, y_{1}\right)
\end{aligned}
$$

one gets $g_{1}=\left(t_{2}-t_{1}\right)\left(t_{3}-t_{2}\right)^{2}$ and $g_{2}=-\left(t_{2}-t_{1}\right)^{2}\left(t_{3}-t_{2}\right)$.

Applying Proposition 3. and some technical manipulations we get the following result:

Proposition 6 [7, Theorem 1] No Bergman polynomials are Lie identities for the Lie algebra so $(4, K, *)$.

The same pattern of proof gives an analogous result concerning $s o(6, K, *)$.

Investigating the identities of minimal degree for the Lie algebras $s o(4), s o(3,1), s o(2,2)$ and $s p(4, R)$ considered in [3] is the next step in research. The physical base of the study in [2] and the common apparatus of doing the investigations both from physical and algebraic point of view are the reason for giving this talk and for the useful discussions made and I hope to be made further for realizing the above mentioned intentions.

\section{References}

[1] G.M. Bergman, Wild automorphisms of free P.I. algebras and some new identities (1981), preprint.

[2] G. Dimitrov, I. Mladenov, A new formula for the exponents of the generators on the Lorentz group, Seventh International Conference on Geometry, Integrability and Quantization Junne 2-10, 2005, Varna, Bulgaria, edited by I. Mladenov \& M. León, SOFTEX, Sofia (2005), 1-18. 
[3] G. Dimitrov, I. Mladenov, On the exponents of some $4 \times 4$ matrices, Proceedings of the Thirty Fifth Spring Conference of the Union of Bulgarian Mathematicians, Borovets, April 5-8 (2006), 152-157.

[4] V. Drensky, Free Algebras and PI-Algebras, Springer, Singapore (1999).

[5] T.G. Rashkova, Bergman type identities in matrix algebras with involution, Proceedings of the Union of Scientists - Rousse, ser. 5 Mathematics, Informatics and Physics 1 (2001), 26-31.

[6] Ts. Rashkova, Identities in algebras with involution, Bull. Austr. Math. Soc. 60 (1999), 469-477.

[7] T.G. Rashkova, Identities in some special Lie algebras, Jubilee Scientific Session, Plovdiv University (2000), 147151.

[8] T.G. Rashkova, The Role of a Theorem of Bergman in Investigating Identities in Matrix Algebras with Symplectic Involution, in "Noncommutative Algebra and Geometry", edited by C. Concini, F. Oystaeyen, N. Vavilov \& A. Yakovlev, A Series of Lecture Notes in Pure and Applied Mathematics, vol.242, Chapman \& Hall/CRC, Taylor \& Francis Group, FL (2006), 175-183.

[9] Ts. Rashkova, V. Drensky, Identities of representations of Lie algebras and *-polynomial identities, Rendiconti del Circolo Matematico di Palermo 48 (1999), 153-162. 